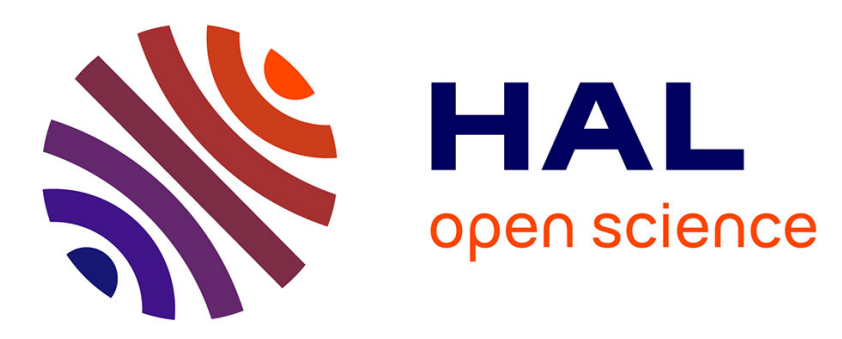

\title{
SINr: Fast Computing of Sparse Interpretable Node Representations is not a Sin!
}

\author{
Thibault Prouteau, Victor Connes, Nicolas Dugué, Anthony Perez, \\ Jean-Charles Lamirel, Nathalie Camelin, Sylvain Meignier
}

\section{- To cite this version:}

Thibault Prouteau, Victor Connes, Nicolas Dugué, Anthony Perez, Jean-Charles Lamirel, et al.. SINr: Fast Computing of Sparse Interpretable Node Representations is not a Sin!. Advances in Intelligent Data Analysis XIX, 19th International Symposium on Intelligent Data Analysis, IDA 2021, Apr 2021, Porto, Portugal. pp.325-337, 10.1007/978-3-030-74251-5_26 . hal-03197434

\section{HAL Id: hal-03197434 \\ https://hal.science/hal-03197434}

Submitted on 14 Apr 2021

HAL is a multi-disciplinary open access archive for the deposit and dissemination of scientific research documents, whether they are published or not. The documents may come from teaching and research institutions in France or abroad, or from public or private research centers.
L'archive ouverte pluridisciplinaire HAL, est destinée au dépôt et à la diffusion de documents scientifiques de niveau recherche, publiés ou non, émanant des établissements d'enseignement et de recherche français ou étrangers, des laboratoires publics ou privés. 


\title{
SINr: fast computing of Sparse Interpretable Node Representations is not a sin!
}

\author{
Thibault Prouteau ${ }^{1}$, Victor Connes ${ }^{2}$, Nicolas Dugué ${ }^{1}$, Anthony Perez ${ }^{3}$, \\ Jean-Charles Lamirel ${ }^{4}$, Nathalie Camelin ${ }^{1}$, and Sylvain Meignier ${ }^{1}$ \\ 1 Le Mans Université, LIUM, EA 4023, Laboratoire d'Informatique de l'Université \\ du Mans, France nicolas.dugue@univ-lemans.fr \\ ${ }^{2}$ Laboratoire des Sciences du Numérique de Nantes, Université de Nantes, France \\ victor. connes@univ-nantes.fr \\ 3 Univ. Orléans, INSA Centre Val de Loire, LIFO EA 4022, FR-45067 Orléans, \\ France anthony.perez@univ-orleans.fr \\ 4 Université de Strasbourg, LORIA, Equipe Synalp, France lamirel@loria.fr
}

\begin{abstract}
While graph embedding aims at learning low-dimensional representations of nodes encompassing the graph topology, word embedding focus on learning word vectors that encode semantic properties of the vocabulary. The first finds applications on tasks such as link prediction and node classification while the latter is systematically considered in natural language processing. Most of the time, graph and word embeddings are considered on their own as distinct tasks. However, word co-occurrence matrices, widely used to extract word embeddings, can be seen as graphs. Furthermore, most network embedding techniques rely either on a word embedding methodology (Word2vec) or on matrix factorization, also widely used for word embedding. These methods are usually computationally expensive, parameter dependant and the dimensions of the embedding space are not interpretable. To circumvent these issues, we introduce the Lower Dimension Bipartite Graphs Framework (LDBGF) which takes advantage of the fact that all graphs can be described as bipartite graphs, even in the case of textual data. This underlying bipartite structure may be explicit, like in coauthor networks. However, with LDBGF, we focus on uncovering latent bipartite structures, lying for instance in social or word co-occurrence networks, and especially such structures providing conciser and interpretable representations of the graph at hand. We further propose SINr, an efficient implementation of the LDBGF approach that extracts Sparse Interpretable Node Representations using community structure to approximate the underlying bipartite structure. In the case of graph embedding, our near-linear time method is the fastest of our benchmark, parameter-free and provides state-ofthe-art results on the classical link prediction task. We also show that low-dimensional vectors can be derived from SINr using singular value decomposition. In the case of word embedding, our approach proves to be very efficient considering the classical similarity evaluation.
\end{abstract}




\section{Introduction}

The aim of graph embedding is to learn representations of nodes encompassing the structural properties of the network. Such representations (or embedding vectors) can then be processed in reduced time and space and proved to be useful to deal with problems such as link prediction [14 and node classification [2]. Among the state-of-the art embedding methods, techniques adapting the Word2vec (for semantic representations of words) framework [15] with random walks have been extensively studied [19, 20]. Matrix factorization approaches [1,6, 17] were also widely considered, some of them also inspired by the GloVe word embedding technique 5. As one can see, natural language processing (NLP) literature influenced a lot the field of graph embedding, providing a solid base on which to build. Indeed, word embedding also aims to provide dense continuous representations, those being used as inputs of NLP systems for opinion mining, translation or text categorization for instance.

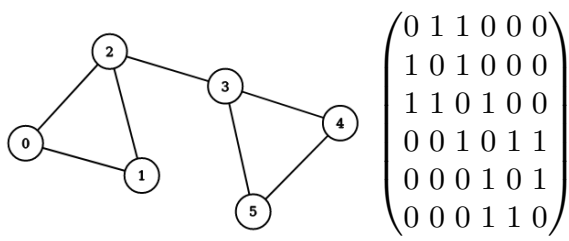

(a)

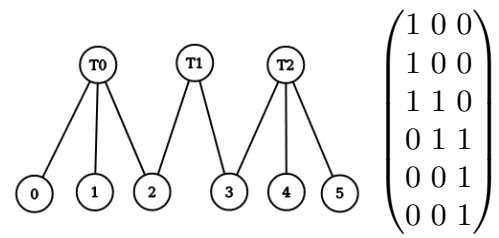

(b)

Fig. 1. Illustrating the LDBGF: (a) a toy graph and its adjacency matrix (b) a lowdimensional bipartite graph representation. The adjacency matrix of the $\perp$ nodes (rows) from the toy graphs are represented with their links to the $T$ nodes (columns). The resulting adjacency matrix is smaller and is actually an interpretable embedding: dimensions represent the connectivity to $\top$ nodes which are tangible entities.

Our approach. To the best of our knowledge, despite the connections between these tasks, graph and word embedding are usually considered as distinct tasks. In this paper, we tackle both of these tasks, considering that word co-occurrence matrices extracted from substantial text corpora can also be seen as graphs. We thus reduce the task of learning word embedding to a graph embedding problem and provide a unified approach for both tasks. Furthermore, in all aforementioned graph embedding works, authors mainly focus on getting efficient and low-dimensional dense vectors, somehow neglecting the time complexity and the interpretability of the results. Meanwhile, interpretability and green computing are major issues for the machine learning field. Taking into account these considerations, we introduce the Lower Dimension Bipartite Graphs Framework (LDBGF), a framework based on low-dimensional bipartite representations of graphs to compute sparse interpretable vectors for words and nodes. 
Guillaume and Latapy [8] showed that all complex networks can be represented by a bipartite structure. Coauthoring networks are by nature represented as a bipartite graph $G=(\top, \perp, E)$ where $\top$ corresponds to the set of papers and $\perp$ to the set of authors, each author being connected to papers they cosigned. The unipartite coauthoring network can thus be retrieved by projecting the bipartite graph, i.e. by adding an edge between any two authors linked to the same paper. When considering social networks, one can reasonably assume that there is a latent bipartite structure 8: people are connected through their school, family, firm, etc. In the case of word co-occurrence networks extracted from large corpora, words are connected by syntactic rules, but also thematic or semantic fields. With LDBGF, we assume that we can uncover low-dimensional latent bipartite structures for real networks, representing them in a concise way (Fig. 11). In such a case, the graph embedding space is interpretable: nodes are represented by their connectivity to the $T$-nodes uncovered that are tangible graph objects (Fig. 1(b)]. Guillaume and Latapy [8 use cliques in the T-part of the bipartite graph to encode relations between nodes. The problem considered can thus be seen as a CLIQUE COVERING problem [8]: given a network, compute a set of cliques such that each edge belongs to at least one clique (ensuring that the $\perp$-projection yields the original network). However, LDBGF enforces the number of cliques to be as small as possible in order to represent the original graph with as few $T$-nodes as possible, and thus get a lower-dimensional embedding space. For instance, considering each edge as a clique would yield too many cliques. Thus, LDBGF is related to MINIMUM CLIQUE COVERING NUMBER, a classical NPHard problem [16. Hence, one needs heuristics to obtain a satisfying covering in a reasonable time.

Our contribution. In line with the LDBGF philosophy, we introduce the SINr 5 (Sparse Interpretable Node Representations) method that derives sparse and interpretable word and graph embedding vectors in near-linear time, thus making a parsimonious usage of CPU resources. The main idea of SINr is to use the community structure to provide an approximate solution to the MINIMUM CLIQUE COVERING problem. Indeed, communities can be seen as clique relaxations that can be uncovered very efficiently [3], allowing SINr to run faster than the other graph embedding approaches. Basing our work on the hypothesis that nodes with similar connectivity to the communities are similar, we compute our bipartite approximation directly from community structure. This is why SINr vectors are sparse and interpretable: each dimension of the embedding vector represents the node connectivity to a community, and the number of dimensions is thus linear in the number of communities. Furthermore, if low-dimensional vectors are required, our SINr vectors can be projected in a lower-dimensional space using standard dimension reduction techniques whilst preserving their efficiency.

Outline. We first describe our SINr embedding technique (Section 1), and how it can be applied to deal with word and graph embeddings. We then detail the

\footnotetext{
5 https://www.github.com/anthonimes/SINr
} 
experimental setup (Section 2), considering graph and textual datasets, and detailing graph and word embedding approaches we compare to. We finally present the very encouraging experimental results on the classical link prediction task for graph embedding, and on the similarity task for word embedding (Section 3). We also demonstrate the lower computational cost of SINr when compared to the other graph embedding methods we consider.

\section{SINr: Algorithmic framework}

SINr is a near-linear time implementation of LDBGF. Since communities can be seen as cliques relaxations [3, instead of relying on cliques to compute a latent bipartite structure (Fig 2(b) , SINr relies on communities which are faster to compute. However, unlike in cliques, nodes connected to a community may not be connected to the whole community. To cope with this problem, we use a recent work by a subset of the same authors, allowing to account for the nodes connectivity to the community structure, the Node F-Measure framework 7 . The aim of our method is to produce embedding vectors where nodes with a similar behaviour towards the community structure of the network lie close in the embedding space. Thus, the approach of SINr actually consists in two steps: the first one is detecting the $p$ communities of the network at hand (Section 1.1). The second, described Section 1.2, is computing the Node Predominance and Node Recall of each node for every community (Fig 2(c) $)$. This allows to obtain a $2 p$ embedding vector for each node (Fig $2(\mathrm{~d})$ ). This explains why SINr leads to sparse and interpretable vectors: dimensions of the embedding space are not abstract but community-related. In the remaining of this section, we describe further the two steps of SINr.

\subsection{First step: community detection algorithms}

We consider (un)directed and (un)weighted graphs $G=(V, E, W)$ with $V$ the set of vertices, $E$ the set of edges and $W$ the weights attached to the edges. The neighbourhood of a vertex $v \in V$ is denoted by $N(v)$, and we let $d(v)=$ $\sum_{u \in N(v)} W_{u, v}$. The community structure of $G$ is a partition $\mathcal{C}=\left\{C_{1}, \ldots, C_{p}\right\}$ of $V$ such that each subgraph $G\left[C_{i}\right]$ is densely connected, while the density between $C_{i}$ and $C_{j}$ is low, $1 \leqslant i<j \leqslant p$. To measure the quality of such a partition, Girvan and Newman $[3]$ introduced the modularity $Q_{\mathcal{C}}$ :

$$
Q_{\mathcal{C}}=\frac{1}{2 m} \cdot \sum_{i, j}\left[A_{i j}-\frac{d_{i} \cdot d_{j}}{2 m}\right] \cdot \delta\left(C_{i}, C_{j}\right)
$$

where $A_{i j}$ denotes the existence (or weight) of edge $i j$, and the $\delta$-function $\delta(u, v)$ is 1 if $u=v$ and 0 otherwise. Computing a partition which maximizes modularity is NP-Hard 4 but there exist community detection algorithms which implement heuristics to maximize modularity, such as the extensively used Louvain algorithm [3]. 
Community structure was already successfully considered to extract graph embedding vectors: Bhowmick et al. 2] used a projection of the hierarchical community tree to obtain the network embeddings. However, our SINr approach relies directly on the community structure, defining the embedding space using the connectivity to the communities. We considered using various algorithms to detect the communities and thus ran several tests using Label Propagation 21] (LP), Infomap, and Louvain algorithms. Both LP and Louvain run fast (nearlinear time), while Infomap is slower but known to perform better. Our experiments have shown that the graph embeddings extracted using Louvain with the SINr methodology perform better for link prediction while the word embeddings extracted with LP perform better on words similarity (Section 3).

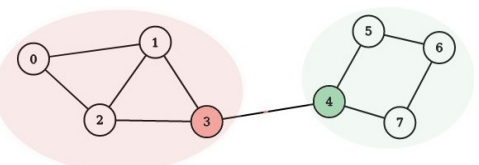

(a)

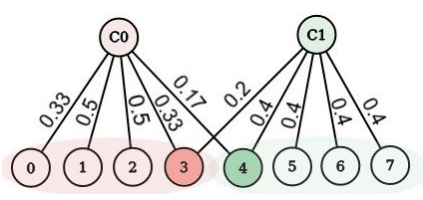

(c)

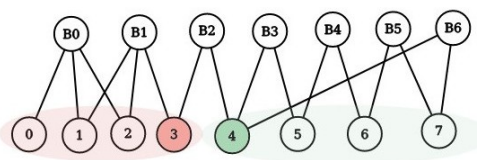

(b)

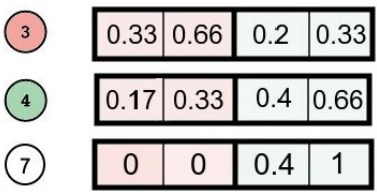

(d)

Fig. 2. SINr : (a) Toy example with oval shapes representing communities (b) Latent bipartite structure using maximum cliques (c) Approximation of such a structure using detected communities and Node predominance (d) SINr vectors with Node predominance and Node recall for both communities.

\subsection{Second step: Node Predominance and Node Recall}

The Node F-measure framework introduced in 7] encompasses interesting topological properties such as centrality and community roles. It is based on Node Predominance (NP) and Node Recall (NR). Node Predominance (Eq. 2 ) is used to characterize the node's connectivity towards its community: the higher it is, the more a node is connected to its community. Node Recall (Eq. 3) is used to evaluate the connectivity of a node with nodes outside its community: the weaker it is, the more the node is connected to the outside. Considering $\mathcal{C}=\left\{C_{1}, \ldots, C_{p}\right\}$, and $1 \leqslant i \leqslant p$, we define NP and NR for each node $u$ as:

$$
\mathrm{NP}_{i}(u)=\frac{d_{C_{i}}(u)}{d_{C_{i}}} \quad(2) \quad \mathrm{NR}_{i}(u)=\frac{d_{C_{i}}(u)}{d(u)}
$$

where $d_{C_{i}}(u)$ is the degree of node $u$ in $C_{i}$, i.e. $d_{C_{i}}(u)=\left|\left\{u v \in E \mid v \in C_{i}\right\}\right|$, and $d_{C_{i}}$ is the number of edges incident to vertices in community $C_{i}$. 
In this work, we consider NP and NR for a given node to any community of a partition. We thus compute $2 p$-dimensional embeddings (Fig $2(\mathrm{~d}))$ that encompass connectivity of the nodes towards the network's communities.

\section{Experimental setup}

\subsection{Datasets}

Networks considered for graph embedding. To conduct our experiments we use several well-known datasets from different fields described by their size $(n=|V|$ and $m=|E|)$. For the sake of our experiments, we extract the largest connected component of each graph and consider them undirected.

(a) Citeseer 13 (Cts, $n=3,312$ and $m=4,660)$ and Cora $13(n=2,708$ and $m=5,278)$ are networks of citations of scientific publications.

(b) Email-eu [11] (Eu, $n=1,005$ and $m=16,706$ ) is a network representing a sender-receiver relationship w.r.t. e-mails within European research institution.

(c) arXiv [9] ( $n=18,771$ and $m=198,050)$ covers scientific collaborations between authors of papers submitted in the Astrophysics category.

(d) Facebook (Fb, $n=63,731$ and $m=817,035)$ represents friendship data of Facebook users.

Corpora considered for word embedding. Our experiments on word embedding span two corpora. For each corpus ${ }^{6}$ words with fewer than four occurrences and stop words (words without semantic interest, e.g. the, at, which) are removed. Furthermore, both corpora are lemmatized using SpaCy.

(a) text8 is made of the first $100 \mathrm{MB}$ of Wikipedia's March 2006 dump. After preprocessing, the corpus contains $6,039,538$ tokens for a vocabulary of 73,860 words.

(b) OANC, the Open American National Corpus is a 15-million-word corpus of written and spoken American English. After preprocessing, the number of tokens of this corpus is $6,016,207$ for a vocabulary of 53,282 words.

\subsection{Extracting word co-occurrence networks for textual corpora}

When dealing with textual data, the first step is to extract a word co-occurrence network from the corpora at hand. To build this graph, in a similar way to other textual word embedding methods 12 , 18, we first compute the word cooccurrence matrix by applying a sliding context window to each sentence in the corpora. At the second step, in order to filter out the insignificant co-occurrences from this matrix, we compute the Pointwise Mutual Information (PMI [12, Eq. 4 for each entry of the matrix, and set entries to 0 when the PMI value is negative. When PMI value is positive, the entry remains unchanged.

\footnotetext{
${ }^{6}$ http://mattmahoney.net/dc/textdata.html and http://www.anc.org/data/oanc/
} 
Let $w_{1}, w_{2}$ be two words, $p\left(w_{1}\right)$ the frequency of $w_{1}$ in the corpora and $p\left(w_{1}, w_{2}\right)$ the co-occurrence frequency of both $w_{1}, w_{2}$. The PMI is defined as follows:

$$
\operatorname{PMI}\left(w_{1}, w_{2}\right)=\log _{2}\left(\frac{p\left(w_{1}, w_{2}\right)}{p\left(w_{1}\right) p\left(w_{2}\right)}\right)
$$

The third step consists in building a weighted graph $G=(V, E, W)$ from the filtered matrix. The vertex set $V$ represents words. The edge set $E$ and the weights $W$ attached to the edges represent the co-occurrences in the corpora: there is an edge between two vertices if these words co-occur significantly in the corpus, and the weight attached to the edge is the number of these cooccurrences.

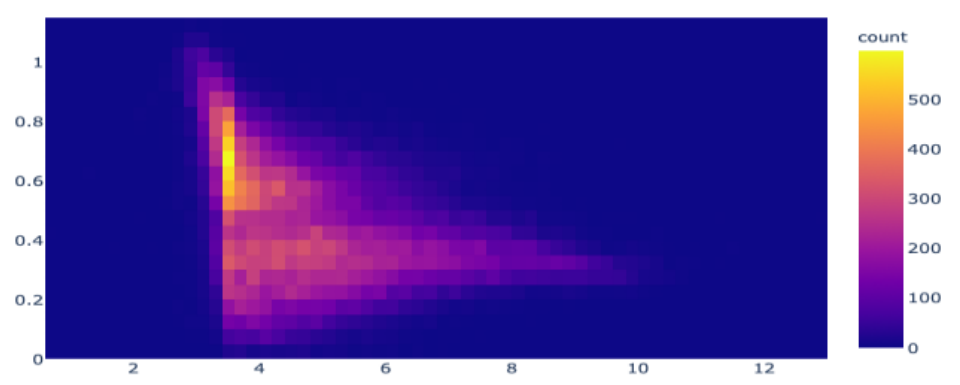

Fig. 3. Heatmap of the weighted degrees of the graph extracted on OANC after IPMI according to the weighted degrees before applying IPMI, abscissa in logarithmic scale.

The final step consists in applying a re-weighting scheme to the graph. According to our extensive experiments, such a process is necessary to extract relevant communities and thus word embeddings. We thus introduce our original IPMI (Iterative Pointwise Mutual Information) re-weighting scheme. Let $E_{\text {ord }}$ be the edges $(u, v)$ of $E$ ordered from the highest to the lowest sum of its weighted degrees $d(u)+d(v)$. For each edge $(u, v)$ ordered as in $E_{\text {ord }}$, we iteratively update $W_{u, v}$ with the IPMI value:

$$
\operatorname{IPMI}(\mathrm{u}, \mathrm{v})=\frac{W_{u, v}}{d(u) d(v)}
$$

To illustrate the effect of the IPMI, we consider the graph extracted from the text8 corpus described Section 2. We plot the weighted degrees of the vertices of this graph after IPMI against the ones before IPMI. As one can see Figure 3 , the plot actually looks like an inverse function. Our experiments thus seem to show that it is important to considerably lower the weights of the hubs (nodes that are widely connected). Our hypothesis is that hubs represent very frequent words, which are more susceptible to be polysemous. It may thus be more relevant to detect communities based on more specific words by lowering the hubs influence before the community detection. 


\subsection{State-of-the-art algorithms}

Graph embedding. We use several state-of-the-art graph embedding techniques provided by the karateclub library 23. to compare with the results obtained by our method. We use the default implementation parameters and embedding vectors dimension number is always set to 128 .

(a) HOPE [17] stands for High-order proximity preserved embedding. This approach uses generalized SVD to approximate proximity matrices such as the ones that can be obtained by using Katz or Adamic-Adar indexes. In our experiments, the proximity matrix used is the Common neighbours one.

(b) Deepwalk (DW) 19 is similar in spirit to the Word2vec Skip-gram approach 15. Instead of computing Skip-gram on sentences, the authors compute it on paths given by random walks on the graph. The number of walks is fixed to 10, the walk length to 80 and the window size to 5 .

(c) Walklets (WL) 20 is also based on random walks, but the authors introduce a sampling method improving the results. The parameters are the same as for Deepwalk except that the window size is 4 .

We also ran experiments with Diff2vec [23, GraRep [6] and LaplacianEigenMaps 1] but for the sake of concision, we do not report their results. Indeed, GraRep is similar to Walklets in spirit, and obtain similar results but it runs slower and requires much more memory. LaplacianEigenmaps and Diff2vec obtain poorer results than the other approaches.

Word embedding. We compare to classical state-of-the-art methods but do not consider contextual word embeddings, since our work focuses on interpretability and parsimonious usage of computing resources. The implementations are detailed below. In all of them, the sliding context window is set to 5 words and the number of dimensions to 300 .

(a) Word2vec (W2V) is one of the most popular methods to learn word embeddings 15. We compute embeddings for each corpus using the CBOW architecture provided by Gensim [22].

(b) GloVe 18] stands for Global Vectors for Word Representation and aims at providing corpus-derived semantic models based on global word co-occurrence statistics. We use the GloVe implementation provided by the authors.

(c) SVD2vec is an implementation 7 of the approach described by Levy et al. 12 using $S V D$ on a PPMI co-occurrence matrix to compute embeddings.

\section{$3 \quad$ Experiments and results}

\subsection{SINr is fast: Complexity and runtime}

Given the community structure of a network, computing embeddings can be done in linear time since one only needs to parse the edges of the graphs to compute

\footnotetext{
7 https://git-lium.univ-lemans.fr/vpelloin/svd2vec
} 
Node Predominance and Node Recall. As stated previously, we use Louvain's algorithm to compute graph embeddings, which is known to run in $O(m)$ time 2, 24. For word embedding, we use Label propagation which is known to run in near-linear time, each propagation step running in $O(m)$ time and the algorithm converging in few steps. Altogether, the running time of our method is thus in $O(m)$. To conclude this section, we present the average tim $8^{8}$ (50 runs) needed to compute embeddings for SINr and graph embedding algorithms HOPE, Deepwalk and Walklets we compare to (see Section 2.3 for more details). As detailed Section 2.1, the Facebook graph contains 63,731 vertices and 817, 035 edges. As one can see, our method is significantly faster than the other approaches.

Table 1. Average runtime in seconds (left) and CPU time taking into account parallelism (right)

\begin{tabular}{cccccc}
\hline & Cora & $\mathrm{Eu}$ & Cts & arXiv & Fb \\
\hline SINr & $\mathbf{0 . 3} / \mathbf{1 . 3}$ & $\mathbf{0 . 3 / 2}$ & $\mathbf{0 . 1} / \mathbf{0 . 9}$ & $\mathbf{0 . 9} / \mathbf{4}$ & $\mathbf{3 / 8}$ \\
HOPE & $0.2 / 3$ & $0.6 / 8$ & $0.7 / 2$ & $10 / 120$ & $26 / 195$ \\
DW & $24 / 36$ & $13 / 18$ & $20 / 30$ & $264 / 378$ & $336 / 422$ \\
WL & $26 / 38$ & $12 / 18$ & $24 / 36$ & $261 / 365$ & $475 / 652$ \\
\hline
\end{tabular}

\subsection{Graph embedding: Link Prediction}

Problem description. Let $G=(V, E)$ be a simple undirected network. Let $U$ denote the universal set containing $\frac{n(n-1)}{2}$ possible pairs of $V$ and $\bar{E}=U \backslash E$ the set of non-edges of $G$. We consider link prediction as a binary classification problem where we assume that there are some missing links (or links that will appear later on) and we train a classifier to detect such links with high probability.

As in [17, we randomly separate the graph into a training set (containing $80 \%$ edges) and a test set containing the remaining edges. We train the embedding vectors on the training set, and then evaluate link prediction on the test set. Training is done using Xgboost and Logistic regression and the best results are kept in each case. For the sake of comparison, we consider the node representation algorithms detailed Section 2.3 with Hadamard product, as well as the following set of heuristic features which is proved to be efficient on the link prediction task (see [14]): Common Neighbours, Adamic Adar, Preferential Attachment, Jaccard index, Resource Allocation Index. The vector obtained from such features is referred to as the heuristics vector.

Evaluation. We consider two evaluation procedures. In the first one, the test set is made of the $20 \%$ of existing edges augmented with the same number of negative examples sampled from $\bar{E}$. We run each test 50 times and present the

\footnotetext{
${ }^{8}$ With two Intel Xeon CPU E5-2660 2.20GHz : 16 cores, 96Go Ram.
} 
averaged accuracy. SINr achieves the highest accuracy for most of the small datasets as one can see Table 2, On sizeable graphs, results are comparable to those of the other state-of-the-art approaches. On such graphs, the communities may be harder to detect, explaining the results. Furthermore, we can observe that results are still close to the best ones, even when applying SVD on SINr in order to get 20 dimensions. Our approach can thus provide both sparse interpretable and dense low-dimensional vectors.

\begin{tabular}{|c|c|c|c|c|c|}
\hline & SINr & INr-SVD & |Heuristics & DW & HOPE \\
\hline Cora & 0.83 & 0.83 & 0.76 & 0.730 .82 & 0.75 \\
\hline $\mathrm{Eu}$ & 0.88 & 0.88 & 0.87 & 0.810 .87 & 0.87 \\
\hline Cts & 0.88 & 0.86 & 0.78 & $\begin{array}{lll}0.76 & 0.87\end{array}$ & 0.83 \\
\hline $\operatorname{arXiv}$ & 0.92 & 0.90 & 0.97 & 0.920 .96 & 0.92 \\
\hline $\mathrm{Fb}$ & 0.91 & 0.89 & 0.93 & 0.860 .92 & 0.90 \\
\hline
\end{tabular}

Table 2. Accuracy on the link prediction task. SINr-SVD is 20- $d$ SINr vectors obtained using $S V D$.

\begin{tabular}{lccc}
\hline & $p$ & $Q_{\mathcal{C}}$ & $\sigma$ \\
\hline Cora & 23 & 0.80 & 0.94 \\
Eu & 6 & 0.41 & 0.44 \\
Cts & 33 & 0.85 & 0.96 \\
arXiv & 28 & 0.62 & 0.87 \\
Fb & 73 & 0.62 & 0.96
\end{tabular}

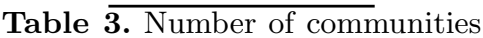
$p$, modularity $Q_{\mathcal{C}}$, and sparsity coefficient $\sigma$ of the SINr vectors.

In our second evaluation procedure, we consider a test set with many more negative examples. Due to the high cardinality of the set $\bar{E}$, we randomly sample about $1 \%$ of such pairs for evaluation on most graphs, except for Facebook where we sample $0.1 \%$. We run each test 50 times and present the averaged precision $₫$. The latter measures the fraction of node pairs in the top- $k$ most probable pairs (according to our classifier) corresponding to an actual edge in the network. Figure 4 presents representative results. For most datasets introduced Section 2.1, experiments highlight the relevance of our method that competes with the state-of-the art approaches. On Email-eu, the lower modularity of the partitions returned (Table 3 degrades the performances of our approach. Our method obtains these encouraging results being by far the fastest (Table 1).
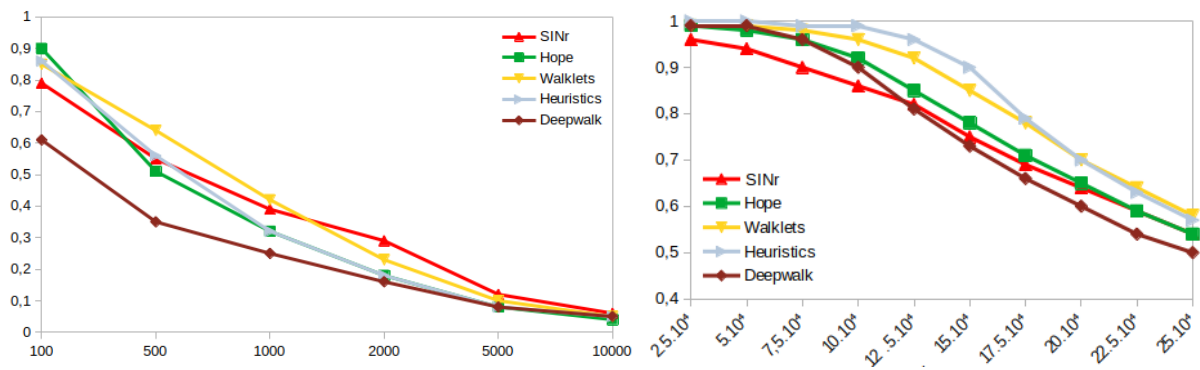

Fig. 4. precision $@$, the proportion among the $k$ most probable pairs that are edges according to the classifier trained on link prediction on Cts (at left) and Fb (at right). 


\subsection{Word embedding: Similarity}

To evaluate the performances of SINr word embeddings, we consider a word similarity task with datasets presented in Lastra-Diaz et al. 10]. Each dataset is composed of pairs of words associated with a similarity value assessed by humans. The evaluation consists in computing the Spearman correlation between the cosine similarity for all the pairs in the embedding space and the human value.

\begin{tabular}{lcccc}
\hline text8 & W2V & GloVe & SVD2vec SINr \\
\hline MC28 & 0.58 & 0.42 & 0.67 & $\mathbf{0 . 6 9}$ \\
RG65 & 0.52 & 0.48 & 0.57 & $\mathbf{0 . 6 4}$ \\
MTurk771 & $\mathbf{0 . 5 2}$ & 0.48 & 0.45 & 0.48 \\
WS353Rel & 0.47 & 0.44 & 0.46 & $\mathbf{0 . 5 0}$ \\
WS353Full & $\mathbf{0 . 5 5}$ & 0.47 & $\mathbf{0 . 5 5}$ & 0.53 \\
MEN & 0.53 & 0.48 & $\mathbf{0 . 6 1}$ & 0.52 \\
\hline
\end{tabular}

\begin{tabular}{lcccc}
\hline OANC & W2V & GloVe & SVD2vec & SINr \\
\hline MC28 & 0.45 & 0.54 & 0.33 & $\mathbf{0 . 6 2}$ \\
RG65 & 0.33 & 0.32 & 0.32 & $\mathbf{0 . 3 9}$ \\
MTurk771 & $\mathbf{0 . 4 4}$ & 0.39 & 0.36 & 0.37 \\
WS353Rel & 0.40 & 0.34 & $\mathbf{0 . 4 7}$ & 0.41 \\
WS353Full & 0.49 & 0.40 & $\mathbf{0 . 5 1}$ & 0.44 \\
MEN & 0.44 & 0.46 & $\mathbf{0 . 5 9}$ & 0.40 \\
\hline
\end{tabular}

Table 4. Word similarity evaluation in Spearman correlation between human judgement and cosine similarity for each model. At left, on text8 corpus, at right on OANC.

The first four datasets (MC28, RG65, MTurk771and WS353Re1) are composed of pairs of names, the last two datasets (WS353Full, MEN) contain names, adjectives and verbs. On both text8 and OANC, SINr word embeddings perform best on MC28 and RG65 whose words in pairs have a similar meaning. W2V and SVD2vec achieve better results than SINr on datasets with related pairs of words (MTurk771 and WS353Rel) and datasets with names adjectives and verbs (WS353Full, MEN). Overall, the performances of SINr on this task remain encouraging and show that word embeddings extracted from co-occurrence networks can achieve good results on the similarity task.

\section{Conclusion}

We introduced LDBGF, a novel approach based on the underlying bipartite structure of networks to compute sparse interpretable embeddings. Moreover, we developed SINr, a near linear-time implementation of this newly introduced framework that is faster than all the other graph embedding approaches we compare to. Although embedding techniques explicitly aim to get dense low-dimensional vectors, we demonstrate that the SINr vectors achieve state-of-the-art results on classic graph and word embedding evaluation tasks whilst maintaining a sparse and interpretable representation. We also show that projecting our embedding vectors into a low-dimensional space using SVD does not significantly lower the results. These results demonstrate the relevance of the LDBGF philosophy. We thus hope for new efficient implementations of this framework. For instance, it would be interesting to extend our approach to deal with temporal networks by considering incremental clustering algorithms. 


\section{References}

1. Belkin, M., Niyogi, P.: Laplacian eigenmaps and spectral techniques for embedding and clustering. In: NIPS. pp. 585-591 (2002)

2. Bhowmick, A.K., Meneni, K., Danisch, M., Guillaume, J., Mitra, B.: Louvainne: Hierarchical louvain method for high quality and scalable network embedding. In: WSDM. pp. 43-51 (2020)

3. Blondel, V.D., Guillaume, J.L., Lambiotte, R., Lefebvre, E.: Fast unfolding of communities in large networks. J. Stat. Mech.: Theory Exp. 2008(10), P10008

4. Brandes, U., Delling, D., Gaertler, M., Görke, R., Hoefer, M., Nikoloski, Z., Wagner, D.: Maximizing modularity is hard. arXiv preprint physics/0608255 (2006)

5. Brochier, R., Guille, A., Velcin, J.: Global vectors for node representations. In: WWW. pp. 2587-2593 (2019)

6. Cao, S., Lu, W., Xu, Q.: Grarep: Learning graph representations with global structural information. In: CIKM. pp. 891-900 (2015)

7. Dugué, N., Lamirel, J.C., Perez, A.: Bringing a feature selection metric from machine learning to complex networks. In: Complex networks (2). pp. 107-118 (2018)

8. Guillaume, J.L., Latapy, M.: Bipartite graphs as models of complex networks. Physica A 371(2), 795-813 (2006)

9. Kunegis, J.: The koblenz network collection. In: WWW. pp. 1343-1350 (2013)

10. Lastra-Díaz, J.J., Goikoetxea, J., Hadj Taieb, M.A., García-Serrano, A., Aouicha, M.B., Agirre, E.: Reproducibility dataset for a large experimental survey on word embeddings and ontology-based methods for word similarity. Data in Brief 26, $104432(2019)$

11. Leskovec, J., Kleinberg, J., Faloutsos, C.: Graph evolution: Densification and shrinking diameters. ACM Trans. Kno. Discov. from Data 1(1), 2-es (2007)

12. Levy, O., Goldberg, Y., Dagan, I.: Improving distributional similarity with lessons learned from word embeddings. ACL 3, 211-225 (2015)

13. Lu, Q., Getoor, L.: Link-based classification. In: ICML. pp. 496-503 (2003)

14. Martínez, V., Berzal, F., Talavera, J.C.C.: A survey of link prediction in complex networks. ACM Comput. Surv 49(4), 69:1-69:33 (2017)

15. Mikolov, T., Sutskever, I., Chen, K., Corrado, G.S., Dean, J.: Distributed representations of words and phrases and their compositionality. In: NIPS. pp. 3111-3119 (2013)

16. Monson, S.D., Pullman, N.J., Rees, R.: A survey of clique and biclique coverings and factorizations of $(0,1)$-matrices. Bull. Inst. Combin. Appl 14, 17-86 (1995)

17. Ou, M., Cui, P., Pei, J., Zhang, Z., Zhu, W.: Asymmetric transitivity preserving graph embedding. In: SIGKDD. pp. 1105-1114 (2016)

18. Pennington, J., Socher, R., Manning, C.D.: Glove: Global vectors for word representation. In: EMNLP. pp. 1532-1543 (2014)

19. Perozzi, B., Al-Rfou, R., Skiena, S.: Deepwalk: Online learning of social representations. In: SIGKDD. pp. 701-710 (2014)

20. Perozzi, B., Kulkarni, V., Chen, H., Skiena, S.: Don't walk, skip! online learning of multi-scale network embeddings. In: ASONAM. pp. 258-265 (2017)

21. Raghavan, U.N., Albert, R., Kumara, S.: Near linear time algorithm to detect community structures in large-scale networks. Phys review E 76(3), 036106 (2007)

22. Řehůřek, R., Sojka, P.: Software Framework for Topic Modelling with Large Corpora. In: LREC. pp. 45-50 (2010)

23. Rozemberczki, B., Kiss, O., Sarkar, R.: An api oriented open-source python framework for unsupervised learning on graphs (2020)

24. Traag, V.A.: Faster unfolding of communities: Speeding up the louvain algorithm. Physical Review E 92(3), 032801 (2015) 